\title{
man \\ Biomass-Derived Carbon/Sulfur Composite Cathodes with Multiwalled Carbon Nanotube Coatings for Li-S Batteries
}

\author{
Lina Han ${ }^{1}$, Zemin $\mathrm{Li}^{1}$, Yang Feng ${ }^{2}$, Lijiang Wang ${ }^{1,2, *}$, Bowen $\mathrm{Li}^{1}{ }^{1}$, Zijie Lei ${ }^{1}$, Wenyan Wang ${ }^{1}$ \\ and Weiwei Huang ${ }^{3, *}$
}

check for

updates

Citation: Han, L.; Li, Z.; Feng, Y.; Wang, L.; Li, B.; Lei, Z.; Wang, W.; Huang, W. Biomass-Derived Carbon/Sulfur Composite Cathodes with Multiwalled Carbon Nanotube Coatings for Li-S Batteries. Processes 2022, 10, 136. https://doi.org/ $10.3390 /$ pr10010136

Academic Editor: Antonio Bertei

Received: 12 September 2021 Accepted: 27 December 2021 Published: 10 January 2022

Publisher's Note: MDPI stays neutral with regard to jurisdictional claims in published maps and institutional affiliations.

Copyright: (C) 2022 by the authors. Licensee MDPI, Basel, Switzerland. This article is an open access article distributed under the terms and conditions of the Creative Commons Attribution (CC BY) license (https:// creativecommons.org/licenses/by/ $4.0 /)$
1 College of Chemical Engineering, Hebei Normal University of Science and Technology, Qinhuangdao 066600, China; hanlina0522@gmail.com (L.H.); lizemin1212@gmail.com (Z.L.); libowen0226@hotmail.com (B.L.); leizijie233@hotmail.com (Z.L.); wenyan0502@hotmail.com (W.W.)

2 Renewable Energy Conversion and Storage Center (RECAST), Key Laboratory of Advanced Energy Materials Chemistry (Ministry of Education), Engineering Research Center of High-Efficiency Energy Storage (Ministry of Education), College of Chemistry, Nankai University, Tianjin 300071, China; allenyoungfy@gmail.com

3 School of Environmental and Chemical Engineering, Yanshan University, Qinhuangdao 066004, China

* Correspondence: wanglijiang3584@hevttc.edu.cn (L.W.); huangweiwei@ysu.edu.cn (W.H.)

\begin{abstract}
Lithium sulfur (Li-S) batteries stand out among many new batteries for their high energy density. However, the intermediate charge-discharge product dissolves easily into the electrolyte to produce a shuttle effect, which is a key factor limiting the rapid development of Li-S batteries. Among the various materials used to solve the challenges related to pure sulfur cathodes, biomass derived carbon materials are getting wider research attention. In this work, we report on the fabrication of cathode materials for Li-S batteries based on composites of sulfur and biomass-derived porous ramie carbon ( $R C)$, which are coated with multiwalled carbon nanotubes (MWCNTs). RC can not only adsorb polysulfide in its pores, but also provide conductive channels. At the same time, the MWCNTs coating further reduces the dissolution of polysulfides into the electrolyte and weakens the shuttle effect. The sulfur loading rate of RC is $66.3 \mathrm{wt} . \%$. As a result, the initial discharge capacity of the battery is $1325.6 \mathrm{mAh} \cdot \mathrm{g}^{-1}$ at $0.1 \mathrm{C}$ long cycle, and it can still maintain $812.5 \mathrm{mAh} \cdot \mathrm{g}^{-1}$ after 500 cycles. This work proposes an effective double protection strategy for the development of advanced Li-S batteries.
\end{abstract}

Keywords: Li-S batteries; ramie carbon; multiwalled carbon nanotube coating; shuttle effect

\section{Introduction}

With the growing energy crisis and serious global environmental issues, it is urgent to develop renewable and clean energy technologies [1]. In recent years, wind-, solarand hydraulic-based clean energies have attracted increasing research interests. Since these renewable and clean energy sources are intermittent, it is essential to efficiently and economically store electricity generated from such emerging energy sources [2-4].

In the past 30 years, rechargeable lithium-ion batteries have changed our way of life. However, with the rapid development of technology, the state-of-art lithium-ion batteries cannot meet the needs of powering transportation and large-scale energy storage equipment due to their limited energy densities [5]. Since these renewable and clean energy sources are intermittent, it is essential to efficiently and economically store electricity generated from such emerging energy sources. Li-S batteries and aqueous rechargeable zinc-ion batteries (ZIBs) have been considered promising candidates due to their low cost, high safety and environment-friendly characteristics [6,7]. However, Li-S batteries have received more widespread attention due to their high theoretical capacity $\left(1675 \mathrm{mAh} \cdot \mathrm{g}^{-1}\right)$, and high theoretical energy density $\left(2600 \mathrm{Wh} \cdot \mathrm{kg}^{-1}\right)[8,9]$. Nevertheless, Li-S batteries suffer from several drawbacks such as the relatively poor conductivity of sulfur, volume expansion, and most severely, the shuttle effect of lithium polysulfide, which leads to 
the rapid degradation of battery performance during the electrochemical cycling [10-13]. To tackle these problems, various strategies have been developed to optimize the sulfur cathode [14-20]. One of the most common strategies is restraining sulfur to remain at the cathode by adding various carbon materials such as carbon nanofibers, carbon spheres, porous carbon and reduced graphene oxide to the cathode in form of a composite cathode or a cathode coating [21-23]. In this connection, metal organic framework (MOF)/CNTs nanocomposites including that of cobalt (Co) and cerium (Ce) also showed excellent effect as either the sulfur host or the coating interlayer for Li-S batteries [24,25]. Ahn et al. synthesized sulfur-multi-walled carbon nanotubes (S-MWCNTs) composite as a cathode material for Li-S batteries that exhibited excellent performance with high specific capacity and improved cycle durability [26]. However, the preparation process of these carbon additions is complex, and the costs can be high, which impacts their commercial potential. Moreover, most of the reported studies have used commercial materials and it is always attractive to obtain environmentally friendly functional materials from natural organisms due to their relative low cost, simple processing and easy use [27]. Among the various materials used to solve the challenges related to pure sulfur cathodes, biomass derived carbon materials are attractive. Natural biomass porous carbon materials have unique microporous and mesoporous structures, which can effectively bind polysulfides through physical adsorption [28]. Furthermore, many biomass-derived carbon materials contain nitrogen, which can improve the conductivity of carbon sulfur composites and at the same time promote chemical bonding between the carbon support and the sulfur chain in the sulfur loading process. Due to such strong adsorption the shuttle effect can be reduced [29-34]. Recently, biomass silkworm feces-derived porous carbon based three-dimensional conductive cross-linked all-carbon network as a host matrix of sulfur was designed for Li-S batteries by Ren and co-workers which showed good impeding effect for polysulfides dissolution [35]. Similarly, $\mathrm{Li}$, et al. synthesized corncob-derived activated carbon/sulfur as the cathode electrodes for lithium sulfur batteries and the one with low oxygen content showed improved the electrochemical performance [36].

To further weaken the shuttle effect, many researchers have attempted to insert a suitable interlayer between the separator and the cathode to restrain polysulfides and prevent polysulfides from reaching the anode. This strategy successfully slows down the shuttle effect and improves the cycle stability of the battery to a certain extent $[27,37,38]$. However, there is still a gap between the interlayer and the cathode, which leads to the loss of partial polysulfides. Finding a simple method to directly coat the positive electrode surface can avoid the above problems. Thus, it may be a rewarding research strategy to fabricate such interlayers in form of cathode coatings [39,40].

In this study, porous ramie carbon (RC) derived from natural biomass ramie was prepared by a $\mathrm{ZnCl}_{2}$ chemical activation method. The ramie carbon/sulfur composite (RCS) was prepared by melting diffusion. A simple optimization method was used to prepare the MWCNTs positive protective coating. In this composite cathode, the porous structure of RC can effectively bind polysulfides and improve the volume expansion during charge discharge. The MWCNTs coating acts as a "wall" to further decrease the loss of active ingredients from the cathode and reduce the charge transfer resistance $\left(R_{\text {ct. }}\right)$ of the Li-S batteries. The MWCNTs also further reduce the dissolution of polysulfides in the electrolyte, which in turn further attenuates the polysulfide shuttle effect. In this way, the RCS composite cathodes coated with MWCNTs can greatly improve the overall battery performance, which exhibits an initial discharge specific capacity of $1325.0 \mathrm{mAh} \cdot \mathrm{g}^{-1}$ at $0.1 \mathrm{C}$ rate and a maintained $812.5 \mathrm{mAh} \cdot \mathrm{g}^{-1}$ capacity (Calculated by mass of sulfur) after 500 cycles. 


\section{Materials and Methods}

The experimental materials are shown in Table 1.

Table 1. Experimental reagents.

\begin{tabular}{|c|c|c|c|}
\hline Sample & Formula & Providers & Purities \\
\hline Sulfur & S & $\begin{array}{l}\text { Sinopharm Chemical Reagent } \\
\text { Co., Ltd., Shanghai, China. }\end{array}$ & $\mathrm{CP}$ \\
\hline Ramie & - & Tmall, Hangzhou, China. & - \\
\hline Conductive Carbon & $\mathrm{C}$ & Alfa Aesar, Shanghai, China. & AR \\
\hline MWCNTs & $\mathrm{C}$ & Alfa Aesar, Shanghai, China. & AR \\
\hline NMP & $\mathrm{C}_{5} \mathrm{H}_{9} \mathrm{NO}$ & $\begin{array}{l}\text { Sinopharm Chemical Reagent } \\
\text { Co., Ltd., Shanghai, China. }\end{array}$ & AR \\
\hline PVDF & $-\left(\mathrm{CH}_{2}-\mathrm{CF}_{2}\right)_{\mathrm{n}^{-}}$ & $\begin{array}{l}\text { Yuxiang Plastic Material Factory, } \\
\text { Dalian, China. }\end{array}$ & AR \\
\hline Electrolyte & LiTFSI+DME/DOL & $\begin{array}{l}\text { Beijing Institute of Chemical } \\
\text { Reagents, Beijing, China. }\end{array}$ & - \\
\hline
\end{tabular}

\subsection{Synthesis of $R C$}

Ramie fibers were cut into a length of $\approx 5 \mathrm{~mm}$ and were immersed in boiling water for $30 \mathrm{~min}$. The fibers were then washed with deionized water, filtered and dried under vacuum at $100{ }^{\circ} \mathrm{C}$ for $10 \mathrm{~h}$. A $\mathrm{ZnCl}_{2}$ solution of $1.0 \mathrm{~mol} \mathrm{~L}^{-1}$ concentration was added to submerge the dried ramie fibers, the mixture was stirred at $30^{\circ} \mathrm{C}$ for $10 \mathrm{~h}$ and treated with ultrasound for 10 min every $3 \mathrm{~h}$, followed by drying in vacuum at $100^{\circ} \mathrm{C}$ for $10 \mathrm{~h}$. Next, the ramie was annealed in argon atmosphere at $850^{\circ} \mathrm{C}$ for $2 \mathrm{~h}$. Finally, $1.0 \mathrm{~mol} \mathrm{~L}^{-1}$ hydrochloric acid was added to the RC sample, the mixture was stirred with for $4 \mathrm{~h}$, washed to restore $\mathrm{pH}$ neutrality and dried at $100^{\circ} \mathrm{C}$.

\subsection{Preparation of RCS}

RCS was prepared by a simple melt diffusion process. Elemental sulfur (Chemically Pure (CP)) and RC were mixed in a proportion of 3:1, and the mixture was fully ground to achieve a good mixture of the sulfur and RC. The composite material was annealed at $155{ }^{\circ} \mathrm{C}$ for $12 \mathrm{~h}$ in argon flow to allow the molten sulfur to fully penetrate the RC pores. The composite was then heated to $250^{\circ} \mathrm{C}$ and held for $2 \mathrm{~h}$ under Ar atmosphere to evaporate the sulfur accumulated at the sample surface.

\subsection{Preparation of Cathodes with MWCNT Protective Coatings}

The RCS composite was mixed with polyvinylidene fluoride (PVDF, Analytical Reagent (AR)) and conductive carbon (AR) under the mass proportion of 8:1:1. $45 \sim 50 \mu \mathrm{L}$ of Nmethyl pyrrolidone (NMP, AR) were added and the mixture was ground into a slurry, which was poured onto aluminum foil. The composite cathode was obtained after vacuum drying at $60{ }^{\circ} \mathrm{C}$ for $12 \mathrm{~h}$. After weighing and calculation, the sulfur loading in the composite cathode was $0.375 \mathrm{mg} \cdot \mathrm{cm}^{-2}$.

MWCNTs (AR) and PVDF were evenly mixed at a mass ratio of 9:1. $300 \mu \mathrm{L}$ of NMP were added to obtain the slurry. Then, the mixed slurry was put into a defoaming mixer and stirred strongly for $30 \mathrm{~min}$ to obtain a slurry with uniform dispersion. The obtained slurry was coated onto the surface of the cathode to produce a MWCNTs coating of $50 \mu \mathrm{m}$ thickness. The MWCNTs coating cathode was dried at $80^{\circ} \mathrm{C}$ under vacuum for $12 \mathrm{~h}$ and cut into discs of $12 \mathrm{~mm}$ diameter.

\subsection{Material Characterizations}

The phase identification and crystal structure analysis of sulfur, RC and RCS samples were performed by X-ray diffraction (XRD, D/max-2500, Rigaku, Japan). The morphology of RC, RCS and MWCNTs coating RCS samples was investigated by scanning electron microscopy (SEM, SU8010, Hitachi, Tokyo, Japan) and transmission electron microscopy 
(TEM, Tecnai G2 F20 FEI, Hillsborough, OR, USA). At the same time, the thickness of the MWCNTs coating was measured by SEM. The specific surface area and pore size distribution of RC and RCS samples were obtained by measuring Brunauer-Emmett-Teller (BET, NOVA2200e, Quantachrome, Boynton Beach, FL, USA) isotherms. To determine the sulfur content, thermogravimetric analysis (TG, STA 409 PC/PG, NETZSCH, Selbu, Bavaria, Germany) was performed under argon atmosphere from room temperature to $80{ }^{\circ} \mathrm{C}$. X-ray photoelectron spectroscopy (XPS) analysis was carried out using an ESCALAB 250Xi spectrometer (Thermo Fisher, Waltham, MA, USA) using standard $\mathrm{Al} \mathrm{K} \alpha$ radiation.

\subsection{Electrochemical Measurements}

The batteries were assembled by using RCS composite cathodes with $50 \mu \mathrm{m}$ MWCNTs protective coatings, which were labeled "MWCNTs". For comparison, a battery without protective coating of the cathode was assembled and marked as "without-MWCNTs".

The assembly process of LIR 2032 button batteries was carried out in a glove box under argon atmosphere. The negative shell, lithium anode, diaphragm, electrolyte, electrode sheet, gasket, shrapnel and cathode shell were assembled from the bottom to the top. The electrolyte used was $1.0 \mathrm{M}$ lithium bis (trifluoromethanesulfonimide) salt in a solvent mixture of 1,3-dioxolane (DOL) and dimethoxyethane (DME) $(\mathrm{V} / \mathrm{V}=1: 1)$. The diaphragm used is Celgard 2400 with a thickness of $25 \mu \mathrm{m}$. The electrochemical performance of assembled batteries was test after $12 \mathrm{~h}$. Charging-discharging tests were conducted at $0.05 \mathrm{C}$ and $0.1 \mathrm{C}\left(1 \mathrm{C}=1675 \mathrm{~mA} \cdot \mathrm{g}^{-1}\right)$ within a voltage window of $1.4 \sim 2.8 \mathrm{~V}$ using a Land CT2001A battery test system (Wuhan LAND Electronic Co. Ltd., WuHan, China). Cyclic voltammetry $(\mathrm{CV})$ tests were carried out using an electrochemical workstation (CHI600E) with a scanning speed of $0.2 \mathrm{mV} \mathrm{s}^{-1}$ and a scanning range of $1.3 \sim 3.3 \mathrm{~V}$. Electrochemical impedance spectroscopy (EIS) was performed using a CHI600E impedance analyzer over a frequency range of $1 \mathrm{~Hz}-10 \mathrm{MHz}$. The battery assembly and testing process were performed at $25{ }^{\circ} \mathrm{C}$.

\section{Results}

The RC and RCS samples are characterized by TEM, SEM and EDS as demonstrated in Figure 1. The SEM images in Figure 1a,b clearly show a typical RC morphology and well-developed pores. The HRTEM image of the RC sample shown in Figure 1c also shows the porous structure. Figure 1d contains the element mappings of carbon $(\mathrm{C})$, and nitrogen $(\mathrm{N})$, which provided evidence for the occurrence of $\mathrm{N}$ elements in the RC sample. Figure 2 shows the elemental analysis results of the RC sample, where the C content is $94.379 \mathrm{wt} . \%$, and the N content is $5.621 \mathrm{wt} . \%$. The SEM image of the RCS composite is shown in Figure 1e. After the RC is loaded with sulfur through the melt diffusion process, the morphology of the sample changed. The sulfur loading is determined by TG and BET testing. The dark areas observed in the TEM images in Figure $1 \mathrm{f}$ indicate the presence of sulfur particles in the RC pores. The selected area electron diffraction (SAED) image shown in Figure $1 \mathrm{~g}$ is indicative of crystalline sulfur in the RCS composite. To confirm the distribution of carbon, sulfur and nitrogen in the RCS samples, energy dispersive spectroscopy (EDS) element mapping analysis is performed to confirm the uniform dispersion of these elements in the RCS samples (Figure $1 \mathrm{~h}$ ).

In Figure 3a, a schematic drawing of the structure of the MWCNTs coating RCS cathode is presented. Figure $3 \mathrm{~b}$ shows the SEM image of the cathode side with MWCNTs coating. It can be seen that the thickness of the electrode material is $17.1 \mu \mathrm{m}$ and the thickness of the coating is $7.94 \mu \mathrm{m}$. Figure 3c-e illustrates the morphology of the cathode without the MWCNTs coating. It is shown that the cathode surface without protective coating is rather rough, whereas the protective coating of the cathode leads to a network surface structure composed of MWCNTs, and there are few pores on the surface of the coating, which can effectively prevent the active material from dissolving in the electrolyte (Figure 3f-h). The MWCNTs coating structure can be clearly observed from the side of the 
edge (Figure 3g). As magnification is increased, the interlaced carbon nanotubes can be clearly seen (Figure 3 h).

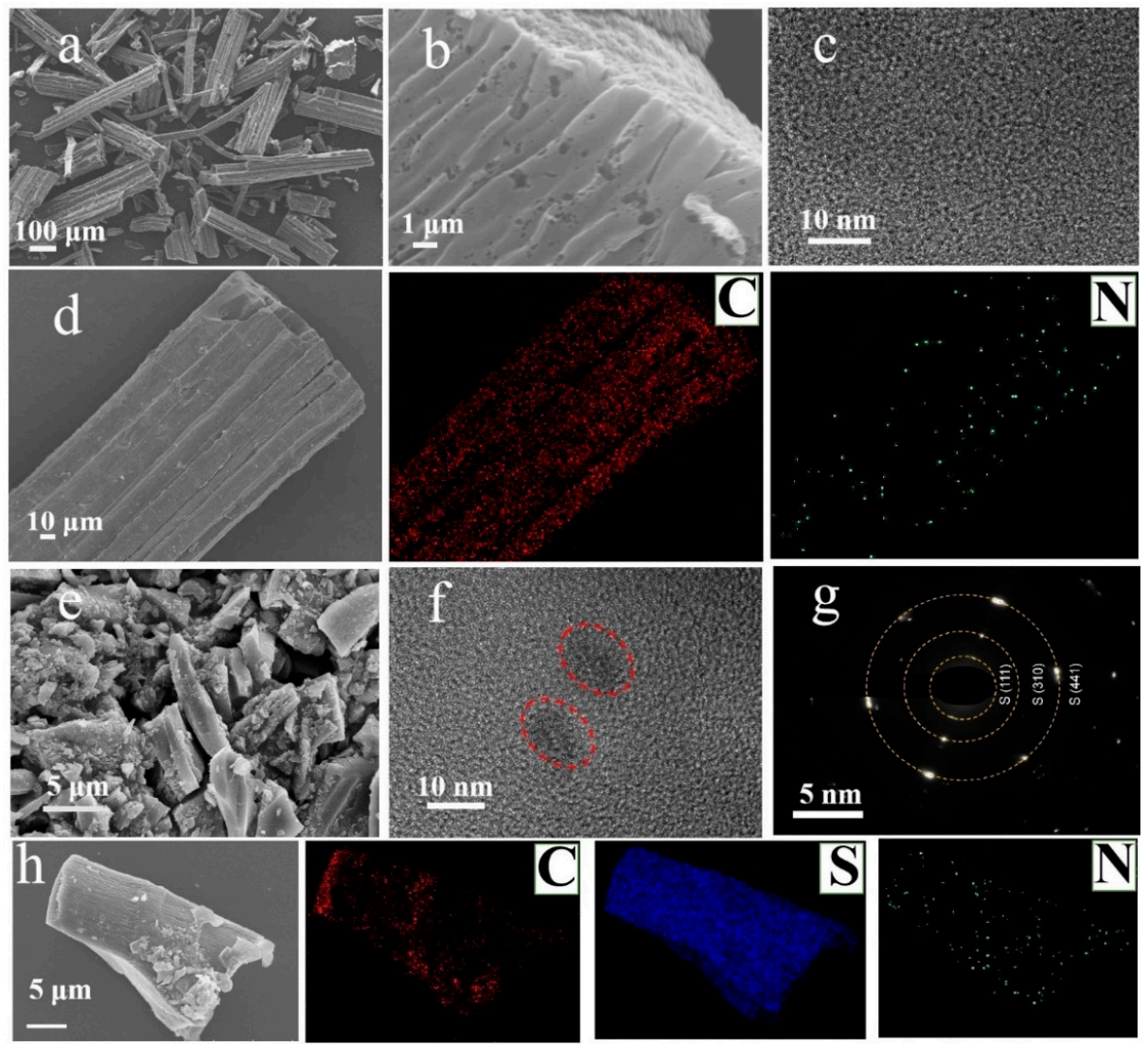

Figure 1. (a,b) SEM images of the RC sample, (c) TEM image of the RC sample, (d) EDS mapping of the RC, (e) SEM image of the RCS, (f) TEM image of the RCS, (g) SAED pattern of the RCS sample and (h) EDS mapping of the RCS.

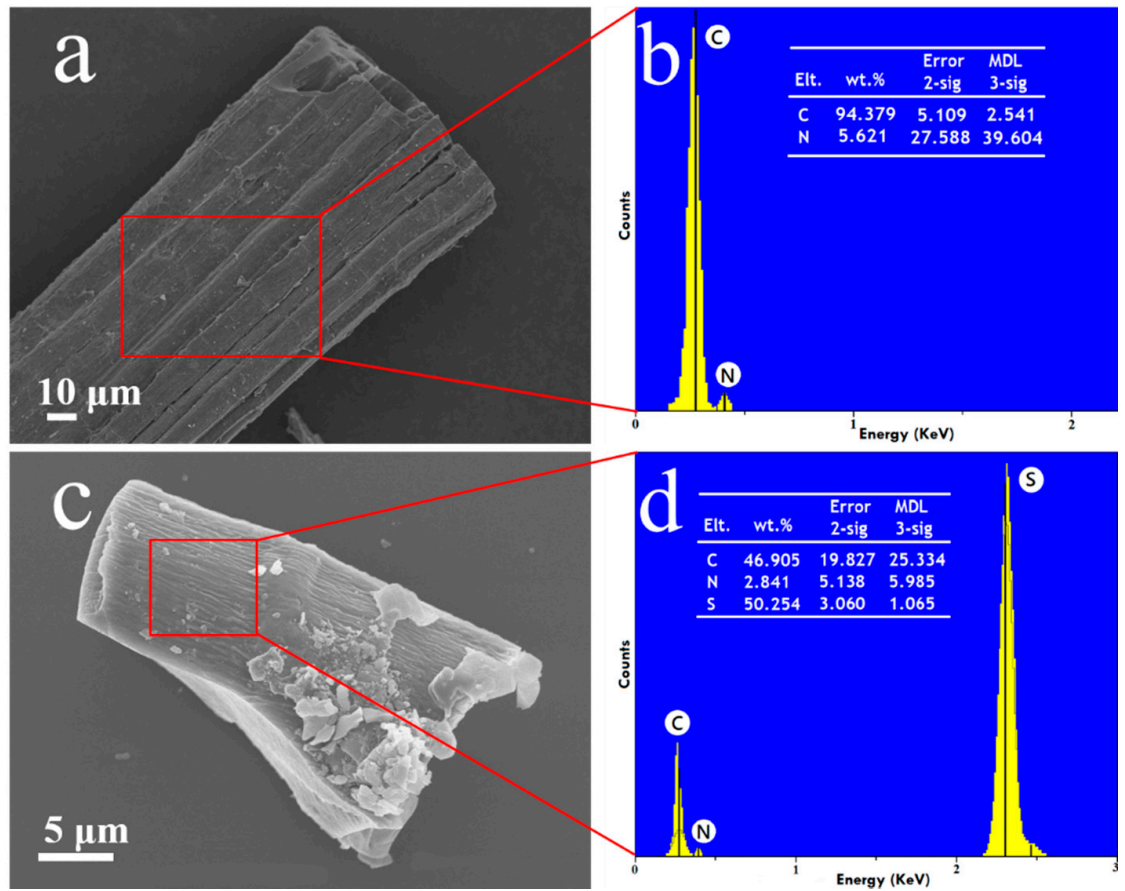

Figure 2. (a) SEM of the RC, (b) EDS of the RC, (c) SEM of the RCS, (d) EDS of the RCS. 
a

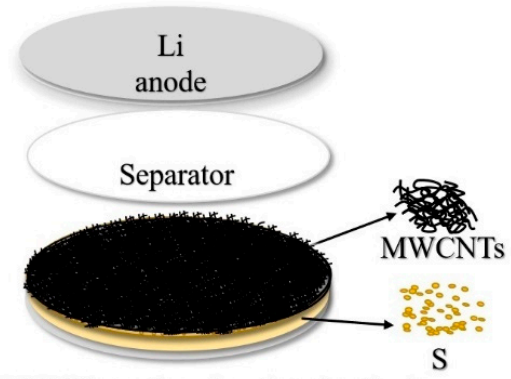

MWCNTs coating-functional cathode
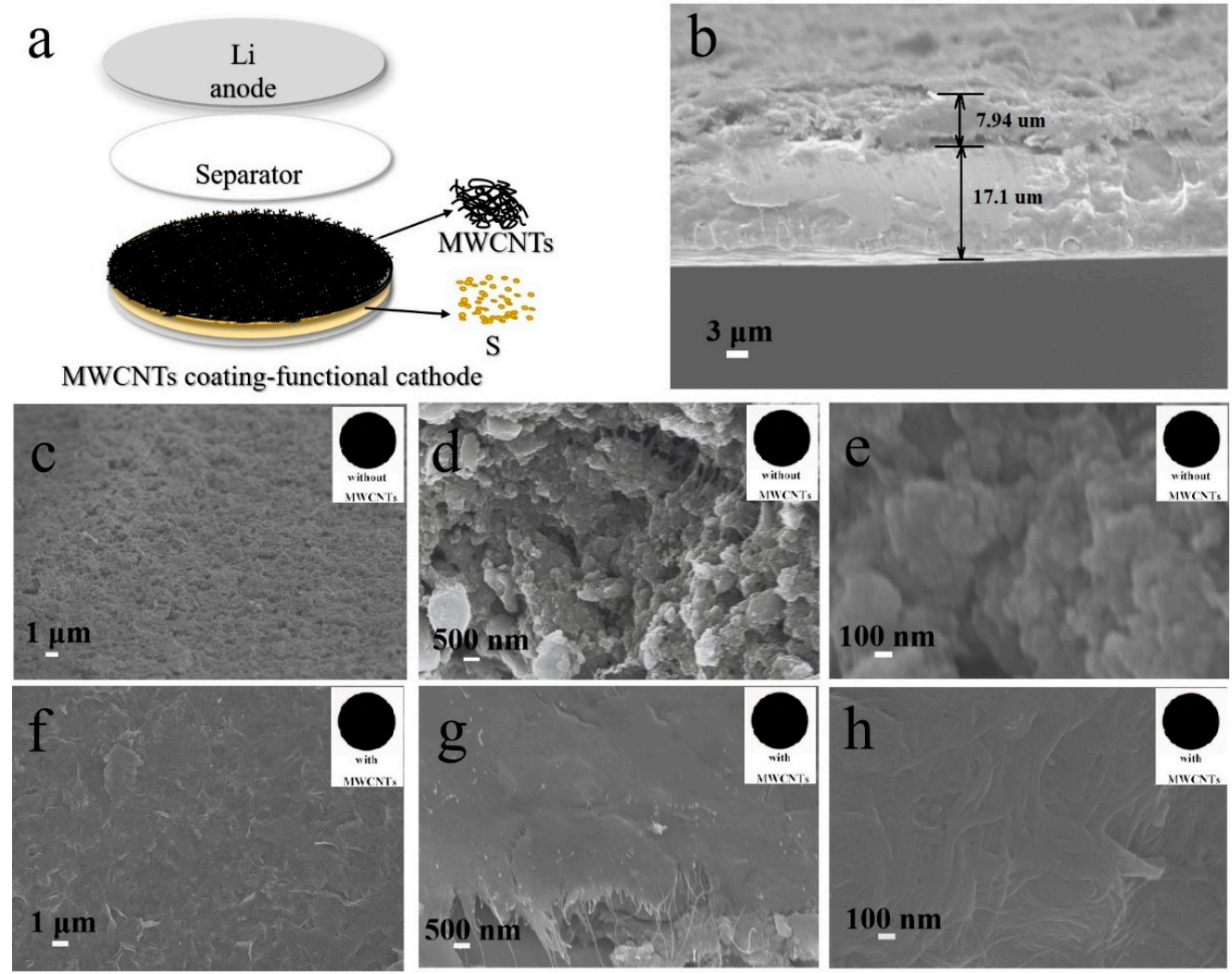

Figure 3. (a) Schematic drawing of the MWCNTs coating cathode, (b) SEM image of the cathode side with MWCNTs coating, (c-e) SEM images of the cathode without MWCNTs coating, (f-h) cathodes with MWCNTs coating.

X-ray photoelectron spectroscopy (XPS) is used to analyze the surface chemical composition of the RCS sample. The XPS results indicate the presence of C, N, S and O atoms on the RCS surface (Figure 4a). The C1s XPS spectrum of the RCS surface (Figure $4 \mathrm{~b}$ ) is composed of four binding energy peaks, which correspond to $\mathrm{C}-\mathrm{C} / \mathrm{C}=\mathrm{C}(284.6 \mathrm{eV}), \mathrm{C}-\mathrm{N}$ $(285.2 \mathrm{eV})$ and $\mathrm{C}=\mathrm{O}(287.7 \mathrm{eV})$ bonds [41]. The high-resolution N1s spectrum of the RCS surface (Figure 4c) shows three peaks located at $398.3 \mathrm{eV}, 399.1 \mathrm{eV}$ and $400.2 \mathrm{eV}$, which correspond to pyridine- $\mathrm{N}$, pyrrolic- $\mathrm{N}$ and graphitic- $\mathrm{N}$ atoms, respectively [42]. No peaks are observed that can be associated with the interaction of N-S bonds. However, previous studies have shown that $\mathrm{N}$-doping in carbon-based materials can improve the affinity between polysulfides and carbon [43]. However, we found that the signal of $\mathrm{N}$ 1s was too weak (but MAPPING proved to contain nitrogen) after many attempts, this may be related to the detection accuracy of our equipment. We will seek more precise and accurate experimental test conditions in our future work.

The XRD patterns of the S, RC and the RCS composite samples are shown in Figure 5a. Characteristic peaks of the RC samples are located at $\approx 22^{\circ}$ and $\approx 44^{\circ}$, which can be associated with carbon giving direct evidence that carbon is present in the samples. A characteristic peak appeared in the RCS composite material, which is associated with sublimated sulfur. This constitutes evidence for presence of crystalline sulfur on the surface of the RCS composites. This may be attributed to sulfur that had settled in large pores that are facing outwards from the sample. 

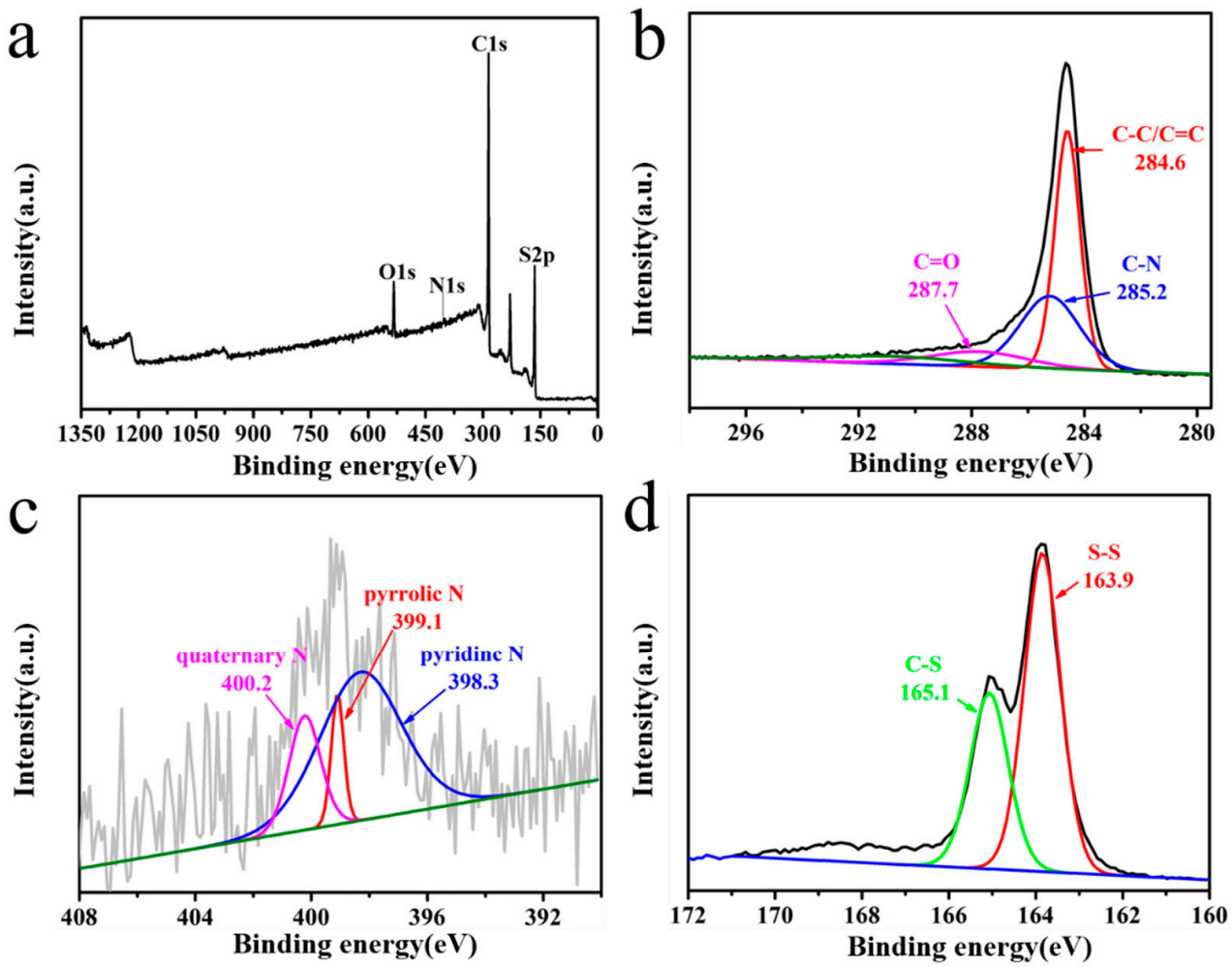

Figure 4. XPS spectrum of RCS composite materials. (a) XPS spectra of surface composition of RCS sample, (b) C1s, (c) N1s, and (d) S2p.
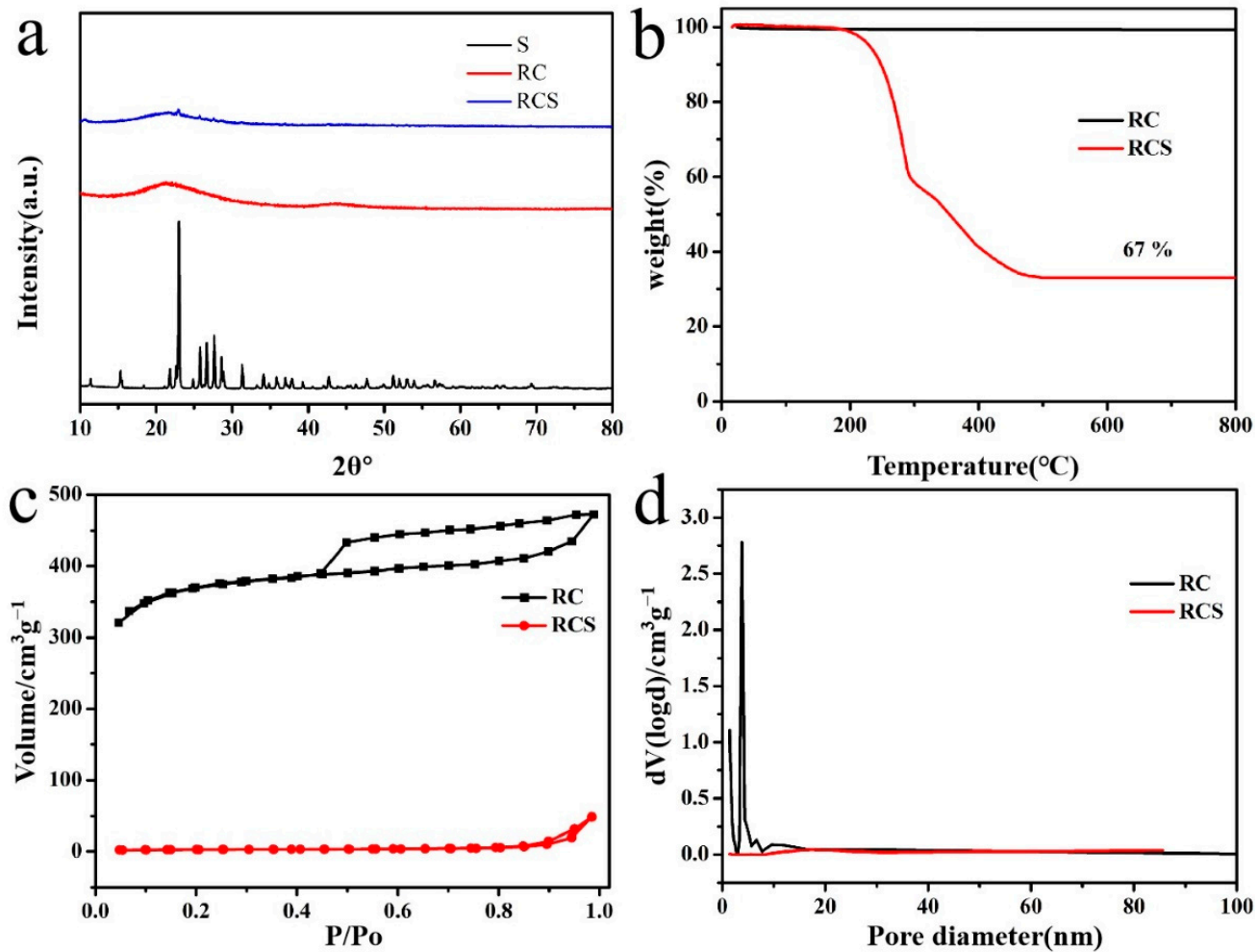

Figure 5. (a) XRD patterns of sulfur, RC and RCS, (b) TG curves of RC and RCS, (c) $\mathrm{N}_{2}$ sorption isotherms of RC and RCS and (d) pore size distributions of RC and RCS.

As mentioned above, RCS is synthesized by a melt diffusion process, where the sulfur content of RCS is determined by TG. Figure $5 b$ shows the TG curve of the RC and RCS 
composite, which is recorded in nitrogen atmosphere from room temperature to $800{ }^{\circ} \mathrm{C}$. The weight loss of RC was only $0.73 \mathrm{wt} . \%$, and the RCS sample had suffered a weight loss of $67 \mathrm{wt} . \%$, the sulfur loading rate of $\mathrm{RC}$ is $66.3 \mathrm{wt} \%$. In the range of $201 \sim 291{ }^{\circ} \mathrm{C}$, the weight loss of RCS composites is large, which may be due to the evaporation of $S$ on the surface of porous carbon or in macropores. At the same time, the weight loss becomes slower after $291^{\circ} \mathrm{C}$, which is due to the insertion of S into the micropores, resulting in a relatively small weight loss.

The pore volume and specific surface area of carbon-based materials are well-known to be two key factors affecting the performance of sulfur-carbon composite cathodes. The high specific surface area helps the sulfur to be better dispersed. Figure $5 c, d$ display the $\mathrm{N}_{2}$ sorption isotherms of RC and RCS. The specific surface area, pore diameter and pore volume of RC are $1329.42 \mathrm{~m}^{2} \cdot \mathrm{g}^{-1}, 3.8 \mathrm{~nm}$ and $0.73 \mathrm{~cm}^{3} \cdot \mathrm{g}^{-1}$, respectively. Compared with $\mathrm{RC}$, the specific surface area and pore volume of RCS decreased to $10.06 \mathrm{~m}^{2} \cdot \mathrm{g}^{-1}$ and $0.07 \mathrm{~cm}^{3} \cdot \mathrm{g}^{-1}$, respectively.

Li-S batteries are assembled using without-MWCNTs and MWCNTs cathodes, which are charged and discharged at a constant voltage in the range of 1.4 2.8 V. The cycle performance of Li-S batteries is tested at $0.1 \mathrm{C}$. After using MWCNTs and RC, the cycle stability of Li-S battery has a certain improvement (Figure 6a). The initial discharge capacities of sulfur electrode made with RC and with MWCNTs (current label "with MWCNTs"), sulfur electrode made with RC but without MWCNTs (current label "without MWCNTs"), sulfur electrode made without RC but with MWCNTs (current label "MWCNTs/S") and sulfur electrode without RC and without MWCNTs (current label "S") are $1325.6 \mathrm{mAh} \cdot \mathrm{g}^{-1}$, $1066.8 \mathrm{mAh} \cdot \mathrm{g}^{-1}, 1045.1 \mathrm{mAh} \cdot \mathrm{g}^{-1}$ and $1042.6 \mathrm{mAh} \cdot \mathrm{g}^{-1}$, respectively. After 500 cycles, the discharge capacity of the with MWCNTs battery is maintained at $812.5 \mathrm{mAh} \cdot \mathrm{g}^{-1}$, the battery without-MWCNTs is maintained at $254.5 \mathrm{mAh} \cdot \mathrm{g}^{-1}$, the battery MWCNTs/S is maintained at $182.3 \mathrm{mAh} \cdot \mathrm{g}^{-1}$ and that of the $\mathrm{S}$ battery decayed to $40.4 \mathrm{mAh} \cdot \mathrm{g}^{-1}$. Obviously, the sulfur battery has the fastest capacity decay, and the battery without-MWCNTs coating modification shows faster capacity decay. This attenuation occurs, because sulfur dissolves in the electrolyte, resulting in the loss of active materials, but the loss of active materials in RCS is even smaller. The MWCNTs coating acted like a wall that could reduce the loss of the active substances. It can be concluded at this point that the dual protection strategy of MWCNTs coating and RC inclusion could significantly improve the overall property of the Li-S batteries.

The rate capacities (Figure $6 \mathrm{c}$ ) of the Li-S batteries are measured at different current rates in the range of $1.4 \sim 2.8 \mathrm{~V}$. First, activate the battery at a rate of $0.05 \mathrm{C}$ for two cycles, and then increase the rate from $0.1 \mathrm{C}$ to $1 \mathrm{C}$ gradually, and then back to $0.1 \mathrm{C}$. Each current density is measured for 10 cycles. The initial specific capacities of MWCNTs, and without-MWCNTs were $1559.3 \mathrm{mAh} \cdot \mathrm{g}^{-1}$ and $1491.5 \cdot \mathrm{mAh}^{-1}$, respectively. When the current density gradually increases, the capacitance gradually decreases. This decrease is smaller for the MWCNTs batteries as compared to the ones without-MWCNTs. When the current density returns to $0.1 \mathrm{C}$, the discharge capacity of MWCNTs batteries returns to $1071.3 \mathrm{mAh} \cdot \mathrm{g}^{-1}$, which indicates that MWCNTs show a better rate performance. Figure $6 \mathrm{~d}$ shows a comparison of the charging-discharging performance of MWCNTs batteries at various rates.

Cyclic voltammetry $(\mathrm{CV})$ tests were performed by using the electrochemical workstation at a scan rate of $0.2 \mathrm{mV} \cdot \mathrm{s}^{-1}$ in the range of $1.3 \sim 3.3 \mathrm{~V}$ (Figure $7 \mathrm{a}, \mathrm{b}$ ). Figure $7 \mathrm{a}, \mathrm{b}$ shows the CV curves of the batteries without and with MWCNTs coatings. Two reduction peaks and an oxidation peak are observed in the CV curve, where both batteries exhibit the typical behavior expected for using sulfur cathodes [44-46]. The batteries with MWCNTs have two oxidation peaks at $2.37 \mathrm{~V}$ and $2.63 \mathrm{~V}$, and two reduction peaks at $2.27 \mathrm{~V}$ and $2.09 \mathrm{~V}$. In the batteries without MWCNTs the two oxidation peaks occur at $2.60 \mathrm{~V}$ and $2.73 \mathrm{~V}$, and the two reduction peaks at $2.22 \mathrm{~V}$ and $1.96 \mathrm{~V}$. These two reduction peaks correspond to the redox reactions of $\mathrm{S}_{8} / \mathrm{Li}_{2} \mathrm{~S}_{\mathrm{x}}$ and $\mathrm{Li}_{2} \mathrm{~S}_{\mathrm{x}} / \mathrm{Li}_{2} \mathrm{~S}$ pairs [47]. The batteries with MWCNTs show lower oxidation and higher reduction peaks. The small polarization of the 
batteries with MWCNTs is due to the strong adsorption capacity and good conductivity of the MWCNTs coating.
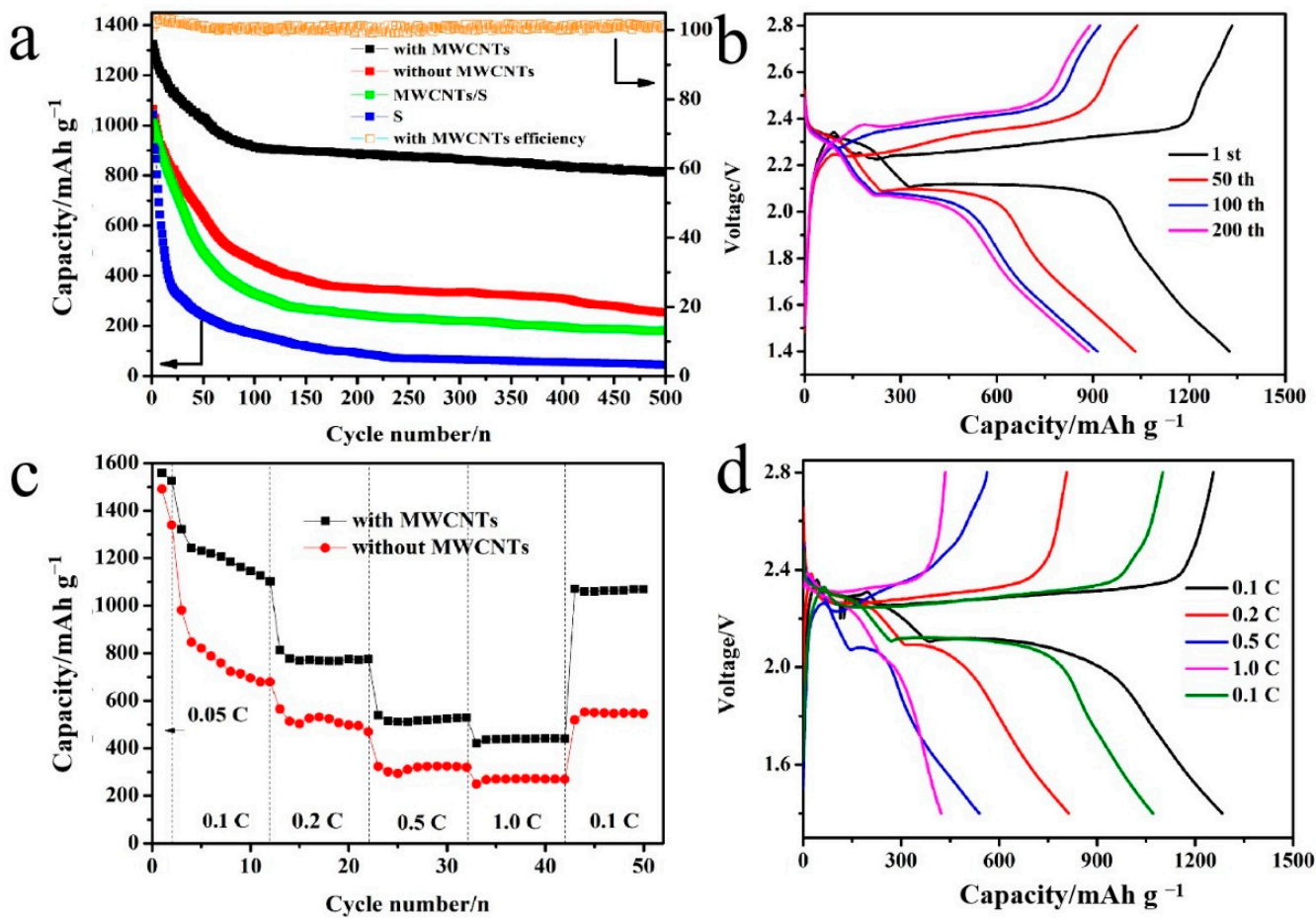

Figure 6. (a) Long-term cycle, (b) galvanostatic discharge-charge voltage profiles for MWCNTs batteries, (c) rate performance graph of Li-S batteries, (d) galvanostatic discharge-charge voltage profiles for MWCNTs batteries at different rates.
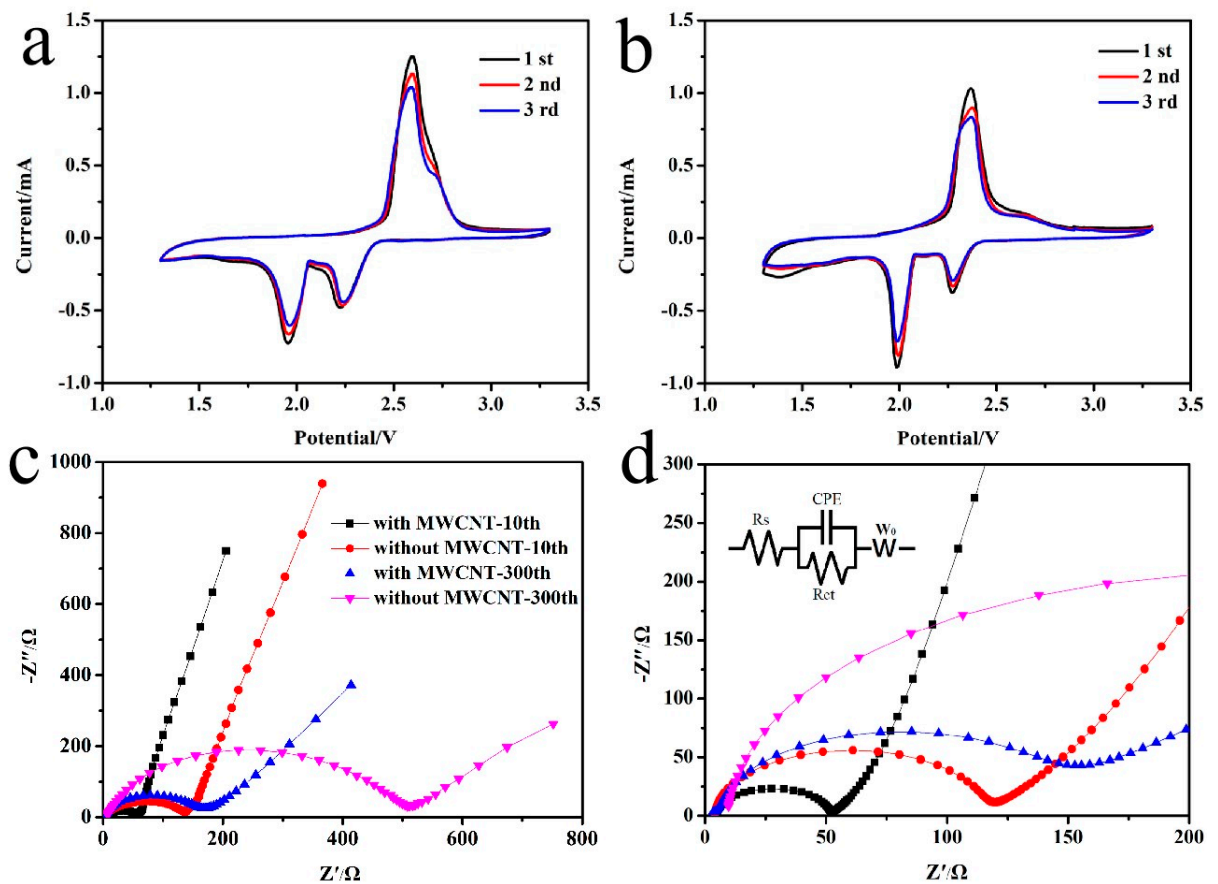

Figure 7. (a) Cyclic voltammogram of batteries without MWCNTs, and (b) with MWCNTs, (c,d) EIS data shown in the format of Nyquist plots of -Z" vs. Z' for Li-S batteries and the equivalent circuit diagram of impedance spectrum. 
Figure 7c shows the EIS Nyquist plots of -Z" vs. Z' after 10 and 300 cycles for batteries without MWCNTs and with MWCNTs. The Nyquist plots display a typical high-frequency semicircle, where the semicircle diameter is related to the charge transfer resistance $R_{\text {ct., }}$, and a low-frequency pike which is indicative of $\mathrm{Li}^{+}$ionic conductivity. Therefore, the smaller the semicircle diameter in the high frequency region, the smaller the resistance of the material [48]. With an increasing number of charge-discharge cycles, the growth of $R_{c t}$. in samples with MWCNTs is significantly smaller than that of uncoated batteries without MWCNTs. The existence of the MWCNTs coating with a network structure greatly reduces the effective resistance of the RCS cathode. These EIS results indicate that the intermediate coating of MWCNTs helps to avoid the loss of the cathode material and promote the electrochemical reaction in the batteries [38]. This article uses the simplified equivalent circuit shown in Figure $7 \mathrm{~d}$ to fit the impedance spectrum. The ultra-high frequency region appears as a point on the impedance spectrum, denoted by Rs. The semicircular curve in the high frequency area is represented by a parallel resistance and a constant phase angle capacitance $\left(\mathrm{R}_{\mathrm{ct}} . / \mathrm{CPE}\right)$, which represents the charge transfer resistance and capacitance in the high frequency area. The oblique line in the low frequency area is represented by a simplified Wo [49].

\section{Conclusions}

In summary, we use $\mathrm{RC}$ prepared by a $\mathrm{ZnCl}_{2}$ chemical activation method as a host material. RCS composites are prepared by a melt diffusion method. MWCNTs coatings are prepared by simply defoaming, stirring and dispersing the slurry directly on the positive surface. Using this type of cathode module is a cost-effective method since ramie biomass is low cost and green. The porous morphology of RC is helpful to the binding of polysulfides and to alleviate the volume expansion during the charge-discharge process. However, due to the inherent problem of the shuttle effect, RCS composite cathodes alone are not capable to achieve high battery capacity and excellent cycle stability. In the design of MWCNTs coating, firstly, the polysulfide can be physically constrained, secondly, the three-dimensional conductive network reduces the impedance and accelerates the reaction. Thanks to this new battery structure, the initial capacity of MWCNTs batteries is $1325.6 \mathrm{mAh} \cdot \mathrm{g}^{-1}$, where the capacity remains high at $812.5 \mathrm{mAh} \cdot \mathrm{g}^{-1}$ after 500 cycles. For comparison, this is $558.0 \mathrm{mAh} \cdot \mathrm{g}^{-1}$ higher than that of the without-MWCNTs. The experimental results indicate that RC addition and MWCNTs coating show a dual function of sulfur fixation, which significantly improves the overall property of the sulfur cathode. This leads to a high discharge specific capacity and cycle stability. The simple, cost effective and environment friendly design provides an effective solution for the "cathode problem" in Li-S batteries.

Author Contributions: Conceptualization, L.W. and W.H.; methodology, L.H., Z.L. (Zemin Li) and B.L.; validation, Z.L. (Zijie Lei) and W.W.; data curation, L.H. and Z.L. (Zemin Li); writing-original draft preparation, L.H.; writing - review and editing, L.W. and Y.F.; funding acquisition, L.W. and W.H. All authors have read and agreed to the published version of the manuscript.

Funding: This work is supported by the National Natural Science Foundation of China (No. 51701049 and 21875206), Hebei Higher Education Institutions of China (Grant No. ZD2016086), Doctoral Research Foundation of Hebei Normal University of Science and Technology (Grant No. 2014YB008).

Conflicts of Interest: The authors declare no conflict of interest.

\section{References}

1. Yu, B.; Chen, Y.; Wang, Z.; Chen, D.; Wang, X.; Zhang, W.; He, J.; He, W. 1T-MoS 2 nanotubes wrapped with N-doped graphene as highly-efficient absorbent and electrocatalyst for Li-S batteries. J. Power Sources 2020, 447, 227364. [CrossRef]

2. Armand, M.; Tarascon, J.-M. Building better batteries. Nature 2008, 451, 652-657. [CrossRef]

3. Wu, F.; Lee, J.T.; Zhao, E.; Zhang, B.; Yushin, G. Graphene-Li 2 S-carbon nanocomposite for lithium-sulfur batteries. ACS Nano 2016, 10, 1333-1340. [CrossRef]

4. Gu, X.; Li, H.; Wen, H.; Zhou, Y.; Kang, H.; Liao, H.; Gao, M.; Wang, Y.; Deng, L.; Yi, X. From agaric hydrogel to nitrogen-doped 3D porous carbon for high-performance Li-S batteries. J. Mater. Sci. 2020, 55, 1136-1147. [CrossRef] 
5. $\mathrm{Gu}, \mathrm{X}$; Lai, C. One dimensional nanostructures contribute better Li-S and Li-Se batteries: Progress, challenges and perspectives. Energy Storage Mater. 2019, 23, 190-224. [CrossRef]

6. $\mathrm{Wu}, \mathrm{T}$.-H.; Liang, W.-Y. Reduced intercalation energy barrier by rich structural water in spinel $\mathrm{ZnMn}_{2} \mathrm{O}_{4}$ for high-rate zinc-ion batteries. ACS Appl. Mater. Interfaces 2021, 13, 23822-23832. [CrossRef]

7. Wu, T.-H.; Lin, W.-S. Boosting proton storage in layered vanadium oxides for aqueous zinc-ion batteries. Electrochim. Acta 2021, 394, 139134. [CrossRef]

8. Li, G.; Lu, F.; Dou, X.; Wang, X.; Luo, D.; Sun, H.; Yu, A.; Chen, Z. Polysulfide regulation by the zwitterionic barrier toward durable lithium-sulfur batteries. J. Am. Chem. Soc. 2020, 142, 3583-3592. [CrossRef] [PubMed]

9. $\quad$ Fang, R.; Zhao, S.; Sun, Z.; Wang, D.W.; Cheng, H.M.; Li, F. More reliable lithium-sulfur batteries: Status, solutions and prospects Adv. Mater. 2017, 29, 1606823. [CrossRef] [PubMed]

10. Zhang, J.; Yang, C.P.; Yin, Y.X.; Wan, L.J.; Guo, Y.G. Sulfur encapsulated in graphitic carbon nanocages for high-rate and long-cycle lithium-sulfur batteries. Adv. Mater. 2016, 28, 9539-9544. [CrossRef] [PubMed]

11. Wang, H.; Zhang, Z.; Wang, Z.; Liang, Y.; Cui, Z.; Zhao, J.; Li, X.; Ren, L. Multistimuli-Responsive microstructured superamphiphobic surfaces with large-range, reversible switchable wettability for oil. ACS Appl. Mater. Interfaces 2019, 11, 28478-28486. [CrossRef] [PubMed]

12. Lang, S.-Y.; Xiao, R.-J.; Gu, L.; Guo, Y.-G.; Wen, R.; Wan, L.-J. Interfacial mechanism in lithium-sulfur batteries: How salts mediate the structure evolution and dynamics. J. Am. Chem. Soc. 2018, 140, 8147-8155. [CrossRef] [PubMed]

13. Zhang, R.; Chi, C.; Wu, M.; Liu, K.; Zhao, T. A long-life Li-S battery enabled by a cathode made of well-distributed B ${ }_{4} \mathrm{C}$ nanoparticles decorated activated cotton fibers. J. Power Sources 2020, 451, 227751. [CrossRef]

14. Lin, J.; Zhang, K.; Zhu, Z.; Zhang, R.; Li, N.; Zhao, C. CoP/C nanocubes-modified separator suppressing polysulfide dissolution for high-rate and stable lithium-sulfur batteries. ACS Appl. Mater. Interfaces 2019, 12, 2497-2504. [CrossRef]

15. Lu, K.; Liu, Y.; Chen, J.; Zhang, Z.; Cheng, Y. Redox catalytic and quasi-solid sulfur conversion for high-capacity lean lithium sulfur batteries. ACS Nano 2019, 13, 14540-14548. [CrossRef]

16. Sevilla, M.; Carro-Rodríguez, J.; Díez, N.; Fuertes, A.B. Straightforward synthesis of Sulfur/N, S-codoped carbon cathodes for Lithium-Sulfur batteries. Sci. Rep. 2020, 12, 2497-2504. [CrossRef]

17. Cai, X.; Cui, B.; Ye, B.; Wang, W.; Ding, J.; Wang, G. Poly (ionic liquid)-Based quasi-solid-state copolymer electrolytes for dynamicreversible adsorption of Lithium polysulfides in Lithium-sulfur batteries. ACS Appl. Mater. Interfaces 2019, 11, 38136-38146. [CrossRef]

18. Ren, Y.; Zeng, L.; Jiang, H.; Ruan, W.; Chen, Q.; Zhao, T. Rational design of spontaneous reactions for protecting porous lithium electrodes in lithium-sulfur batteries. Nat. Commun. 2019, 10, 3249. [CrossRef]

19. Zhao, Z.; Wang, J.; Cheng, M.; Wu, J.; Zhang, Q.; Liu, X.; Wang, C.; Wang, J.; Li, K.; Wang, J. N-doped porous carbon-graphene cables synthesized for self-standing cathode and anode hosts of Li-S batteries. Electrochim. Acta 2020, 349, 136231. [CrossRef]

20. Cha, E.; Patel, M.D.; Park, J.; Hwang, J.; Prasad, V.; Cho, K.; Choi, W. 2D MoS 2 as an efficient protective layer for lithium metal anodes in high-performance Li-S batteries. Nat. Nanotech. 2018, 13, 337-344. [CrossRef]

21. Zhao, Q.; Zhu, Q.; An, Y.; Chen, R.; Sun, N.; Wu, F.; Xu, B. A 3D conductive carbon interlayer with ultrahigh adsorption capability for lithium-sulfur batteries. Appl. Surf. Sci. 2018, 440, 770-777. [CrossRef]

22. Chen, S.-R.; Zhai, Y.-P.; Xu, G.-L.; Jiang, Y.-X.; Zhao, D.-Y.; Li, J.-T.; Huang, L.; Sun, S.-G. Ordered mesoporous carbon/sulfur nanocomposite of high performances as cathode for lithium-sulfur battery. Electrochim. Acta 2011, 56, 9549-9555. [CrossRef]

23. Ji, X.; Lee, K.T.; Nazar, L.F. A highly ordered nanostructured carbon-sulphur cathode for lithium-sulphur batteries. Nat. Mater. 2009, 8, 500-506. [CrossRef]

24. Hong, X.J.; Song, C.L.; Yang, Y.; Tan, H.C.; Li, G.H.; Cai, Y.P.; Wang, H. Cerium based metal-organic frameworks as an efficient separator coating catalyzing the conversion of polysulfides for high performance lithium-sulfur batteries. ACS Nano 2019, 13, 1923-1931. [CrossRef]

25. Sung, S.; Kim, B.H.; Lee, S.; Choi, S.; Yoon, W.Y. Increasing sulfur utilization in lithium-sulfur batteries by a Co-MOF-74@ MWCNT interlayer. J. Energy Chem. 2021, 60, 186-193. [CrossRef]

26. Ahn, W.; Kim, K.B.; Jung, K.N.; Shin, K.H.; Jin, C.S. Synthesis and electrochemical properties of a sulfur-multi walled carbon nanotubes composite as a cathode material for lithium sulfur batteries. J. Power Sources 2012, 202, 394-399. [CrossRef]

27. Jiang, Q.; Zhang, Z.; Yin, S.; Guo, Z.; Wang, S.; Feng, C. Biomass carbon micro/nano-structures derived from ramie fibers and corncobs as anode materials for lithium-ion and sodium-ion batteries. Appl. Surf. Sci. 2016, 379, 73-82. [CrossRef]

28. Benítez, A.; Amaro-Gahete, J.; Chien, Y.C.; Caballero, Á.; Morales, J.; Brandell, D. Recent advances in lithium-sulfur batteries using biomass-derived carbons as sulfur host. Renew. Sust. Energ. Rev. 2022, 154, 111783. [CrossRef]

29. Huang, J.; Lin, Y.; Yu, J.; Li, D.; Du, J.; Yang, B.; Li, C.; Zhu, C.; Xu, J. N-doped foam flame retardant polystyrene derived porous carbon as an efficient scaffold for lithium-selenium battery with long-term cycling performance. Chem. Eng. J. 2018, 350, 411-418. [CrossRef]

30. Yao, M.; Zhu, J.; Meng, W.; Li, C.; Li, C.; Wang, L.; Jiang, Z.; He, Z.; Li, Y.; Meng, W. Enhanced lithium storage performance of nanostructured $\mathrm{NaTi}_{2}\left(\mathrm{PO}_{4}\right)_{3}$ decorated by nitrogen-doped carbon. Electrochim. Acta 2019, 294, 226-232. [CrossRef]

31. Song, J.; Yu, Z.; Gordin, M.L.; Wang, D. Advanced sulfur cathode enabled by highly crumpled nitrogen-doped graphene sheets for high-energy-density lithium-sulfur batteries. Nano Lett. 2016, 16, 864-870. [CrossRef] 
32. Fu, A.; Wang, C.; Pei, F.; Cui, J.; Fang, X.; Zheng, N. Recent advances in hollow porous carbon materials for lithium-sulfur batteries. Small 2019, 15, 1804786. [CrossRef]

33. Yao, Y.; Wu, F. Naturally derived nanostructured materials from biomass for rechargeable lithium/sodium batteries. Nano Energy 2015, 17, 91-103. [CrossRef]

34. Cao, W.; Zhang, E.; Wang, J.; Liu, Z.; Ge, J.; Yu, X.; Yang, H.; Lu, B. Potato derived biomass porous carbon as anode for potassium ion batteries. Electrochim. Acta 2019, 293, 364-370. [CrossRef]

35. Ren, M.; Lu, X.; Chai, Y.; Zhou, X.; Ren, J.; Zheng, Q.; Lin, D. A three-dimensional conductive cross-linked all-carbon network hybrid as a sulfur host for high performance lithium-sulfur batteries. J. Colloid Interface Sci. 2019, 552, 91-100. [CrossRef]

36. Li, B.; Xie, M.; Yi, G.; Zhang, C. Biomass-Derived activated carbon/sulfur composites as cathode electrodes for Li-S batteries by reducing the oxygen content. RSC Adv. 2020, 10, 2823-2829. [CrossRef]

37. Manthiram, A.; Fu, Y.; Chung, S.-H.; Zu, C.; Su, Y.-S. Rechargeable lithium-sulfur batteries. Chem. Rev. 2014, 114, 11751-11787. [CrossRef]

38. Su, Y.-S.; Manthiram, A. A new approach to improve cycle performance of rechargeable lithium-sulfur batteries by inserting a free-standing MWCNT interlayer. Chem. Commun. 2012, 48, 8817-8819. [CrossRef]

39. Huang, J.-Q.; Zhang, Q.; Wei, F. Multi-functional separator/interlayer system for high-stable lithium-sulfur batteries: Progress and prospects. Energy Storage Mater. 2015, 1, 127-145. [CrossRef]

40. Li, Y.; Yuan, L.; Li, Z.; Qi, Y.; Wu, C.; Liu, J.; Huang, Y. Improving the electrochemical performance of a lithium-sulfur battery with a conductive polymer-coated sulfur cathode. RSC Adv. 2015, 5, 44160-44164. [CrossRef]

41. Wang, Q.; Zhang, Y.; Jiang, H.; Meng, C. In-situ grown manganese silicate from biomass-derived heteroatom-doped porous carbon for supercapacitors with high performance. J. Colloid Interface Sci. 2019, 534, 142-155. [CrossRef]

42. Lei, W.; Liu, H.; Xiao, J.; Wang, Y.; Lin, L. Moss-derived mesoporous carbon as bi-functional electrode materials for lithium-sulfur batteries and supercapacitors. Nanomaterials 2019, 9, 84. [CrossRef] [PubMed]

43. Song, J.; Xu, T.; Gordin, M.L.; Zhu, P.; Lv, D.; Jiang, Y.B.; Chen, Y.; Duan, Y.; Wang, D. Nitrogen-doped mesoporous carbon promoted chemical adsorption of sulfur and fabrication of high-areal-capacity sulfur cathode with exceptional cycling stability for lithium-sulfur batteries. Adv. Funct. Mater. 2014, 24, 1243-1250. [CrossRef]

44. Guo, J.; Xu, Y.; Wang, C. Sulfur-Impregnated disordered carbon nanotubes cathode for lithium-sulfur batteries. Nano Lett. 2011, 11, 4288-4294. [CrossRef]

45. Fu, Y.; Manthiram, A. Core-Shell structured sulfur-polypyrrole composite cathodes for lithium-sulfur batteries. RSC Adv. 2012, 2, 5927-5929. [CrossRef]

46. Zhao, M.-Q.; Zhang, Q.; Huang, J.-Q.; Tian, G.-L.; Nie, J.-Q.; Peng, H.-J.; Wei, F. Unstacked double-layer templated graphene for high-rate lithium-sulphur batteries. Nature Commun. 2014, 5, 3410. [CrossRef]

47. Seh, Z.W.; Sun, Y.; Zhang, Q.; Cui, Y. Designing high-energy lithium-sulfur batteries. Chem. Soc. Rev. 2016, 45, 5605-5634. [CrossRef]

48. Yuan, L.; Qiu, X.; Chen, L.; Zhu, W. New insight into the discharge process of sulfur cathode by electrochemical impedance spectroscopy. J. Power Sources 2009, 189, 127-132. [CrossRef]

49. Holzapfel, M.; Martinent, A.; Alloin, F.; Le Gorrec, B.; Yazami, R.; Montella, C. First lithiation and charge/discharge cycles of graphite materials, investigated by electrochemical impedance spectroscopy. J. Electroanal. Chem. 2003, 546, 41-50. [CrossRef] 